\title{
Influence of the equation of state of matter and ion beam characteristics on target heating and compression
}

\author{
N. A. Tahir, ${ }^{1}$ A. Shutov, ${ }^{2}$ D. Varentsov, ${ }^{3}$ P. Spiller, ${ }^{3}$ S. Udrea, ${ }^{4}$ D. H. H. Hoffmann,,${ }^{4,3}$ I. V. Lomonosov, ${ }^{2}$ J. Wieser, ${ }^{3}$ \\ M. Kirk, ${ }^{3}$ R. Piriz, ${ }^{5}$ V. E. Fortov, ${ }^{2}$ and R. Bock $^{3}$ \\ ${ }^{1}$ Institut für Theoretische Physik, Universität Frankfurt, Postfach 11 19 32, 60054 Frankfurt, Germany \\ ${ }^{2}$ Institute of Problems of Chemical Physics, Russian Academy of Sciences, Institutskii pr. 18, 142432 Chernogolovka, Russia \\ ${ }^{3}$ Gesellschaft für Schwerionenforschung, Planckstraße 1, 64291 Darmstadt, Germany \\ ${ }^{4}$ Institut für Kernphysik, Technische Universität Darmstadt, Schlossgartenstraße 9, 64289 Darmstadt, Germany \\ ${ }^{5}$ E.T.S.I. Industriales, Universidad de Castilla-La Mancha, 13071 Ciudad Real, Spain
}

(Received 3 July 2002; published 19 February 2003)

\begin{abstract}
The subject of high-energy density (HED) in matter is of considerable interest to many branches of physics. Intense beams of energetic heavy ions are a promising tool for creating large samples of HED matter which can be used to study the equation-of-state properties of such exotic states of matter experimentally. The Gesellschaft für Schwerionenforschung (GSI), Darmstadt, is a unique laboratory worldwide which has a heavy ion synchrotron facility, SIS18 (with a magnetic rigidity of $18 \mathrm{Tm}$ ), that delivers intense heavy ion beams. Using the beams generated at this present facility, interesting experimental work has been carried out in the field of HED matter [D. H. H. Hoffmann et al., Nucl. Instrum. Methods Phys. Res., Sect. B 161-162, 9 (2000)]. The GSI is planning to significantly expand its accelerator capabilities with construction of a new synchrotron ring, SIS100, which will have a magnetic rigidity of $100 \mathrm{Tm}$. This new facility will deliver a uranium beam which will have orders of magnitude higher intensity than the existing facility and will also have the possibility of multibeam acceleration. This paper presents two-dimensional hydrodynamic simulations of different target geometries including solid as well as hollow cylinders that are irradiated with beams having different shapes of the focal spot which will be available at the SIS100 facility. These include a circular focal spot, an annular focal spot, and an elliptic focal spot, respectively. The purpose of this study is to determine the region of the physical parameters including density, temperature, and pressure that can be accessed using the SIS100 beam. This information, we hope, will be useful for designing experiments on the studies of thermophysical properties of matter including the designing of appropriate diagnostic tools.
\end{abstract}

DOI: $10.1103 /$ PhysRevSTAB.6.020101

PACS numbers: $51.50 .+\mathrm{v}, 51.60 .+\mathrm{a}, 51.70 .+\mathrm{f}$

\section{INTRODUCTION}

The accelerator capabilities of the Gesellschaft für Schwerionenforschung (GSI), Darmstadt, will be significantly enhanced due to construction of a new synchrotron facility, SIS100, which will have a magnetic rigidity of $100 \mathrm{Tm}$. This new facility will deliver a uranium beam with an intensity of $1-2 \times 10^{12}$ ions which will be delivered in a single bunch. A wide range of particle energy ranging from $400 \mathrm{MeV} / \mathrm{u}$ to $2.7 \mathrm{GeV} / \mathrm{u}$ will be available while the corresponding pulse length for the above energy range will be $90-$ $20 \mathrm{~ns}$, respectively. The preliminary design studies show that the beam can be focused to a beam spot with a radius of $1.0 \mathrm{~mm}$. It is to be noted that the beam power profile along the radial direction is considered to be Gaussian and the full width at half maximum (FWHM) of this distribution can be regarded as the beam radius.

Unlike lasers, energetic heavy ions deposit their energy deep into solid matter. Intense heavy ion beams are therefore a suitable and efficient tool for creating large samples of high-energy-density (HED) matter including strongly coupled plasmas. The GSI is a unique laboratory worldwide that has the possibility of generating intense heavy ion beams. The existing heavy ion synchrotron, SIS18 at the GSI, can deliver intense beams of different species of ions. Using the beams generated at the existing heavy ion accelerator facility, SIS18, important experimental work has been done in this field during the past few years [1-5]. Detailed numerical simulations of these experiments were also performed in order to provide the necessary theoretical support. These simulations were carried out using a two-dimensional hydrodynamic computer code, BIG-2 [6].

Using the above computer code, we have done extensive simulation studies of beam-matter heating using the future SIS100 beam parameters and employing different target geometries that may be used in the future experiments. The purpose of this simulation study is to predict the physical conditions that can be achieved in the target by the SIS100 beam. This information we believe will be very useful in designing the future experiments including the development and design of the necessary diagnostic tools. 


\section{FUTURE ACCELERATOR FACILITY AT GSI DARMSTADT}

The GSI Darmstadt has proposed a major upgrade of the existing heavy ion accelerator facility. This includes a substantial increase in the average number of particles, the number of particles per pulse, and improvement in the quality and quantity of the secondary beams for storage ring experiments. The main component of the envisaged facility is a fast cycled, two stage, superconducting heavy ion synchrotron with a maximum magnetic rigidity of $200 \mathrm{Tm}$.

The first stage consists of SIS100 which has a maximum magnetic rigidity of $100 \mathrm{Tm}$. It will be used as a high intensity driver and is able to generate short single bunches of heavy ions, for example, uranium +28 with particle energies of up to $2.7 \mathrm{GeV} / \mathrm{u}$. The second stage, SIS200, will be used as a stretcher ring for slow extraction and for further acceleration of a highly charged uranium beam up to kinetic energies of $23 \mathrm{GeV} / \mathrm{u}$.

The first synchrotron stage, SIS100, can be used as an optimized driver to carry out interesting physics experiments including the creation of high-energy-density matter.

This facility will be equipped with a dedicated $\mathrm{rf}$ system in order to achieve strong pulse compression of beams up to $100 \mathrm{Tm}$. The reference ion species considered in the design are intermediate charged heavy ions such as $\mathrm{U}^{+28}$. The existing accelerators, UNILAC (Universal Linear Accelerator) and heavy ion synchrotron, SIS18, which has a magnetic rigidity of $18 \mathrm{Tm}$, will become the injector chain for the new synchrotron. The SIS18 will be operated as a fast cycled booster synchrotron.

To achieve a maximum number of uranium ions in the new SIS100, a uranium beam with a charge state +4 will be produced using a MEVVA (metal evaporation vacuum arc) source and preaccelerated in the new high current injector. The required beam current before stripping to a charge state +28 (at $1.4 \mathrm{MeV} / \mathrm{u})$ is $15 \mathrm{emA}$. After further acceleration in the ALVAREZ section of the UNILAC, the $\mathrm{U}^{+28}$ beam will be injected at $11.4 \mathrm{MeV} / \mathrm{u}$ in the SIS18. A maximum number of $2.5 \times 10^{11}$ ions can be stacked by means of a multiturn injection and will be accelerated to about $196 \mathrm{MeV} / \mathrm{u}$. In order to minimize beam losses due to ionization of the beam ions as a result of collisions with the residual gas atoms, acceleration in the SIS18 will be performed as fast as possible (at maximum, with a magnetic field ramping speed of $10 \mathrm{~T} / \mathrm{s}$ ).

Before transferring the ion beam to SIS100, an equilibration of vertical and horizontal phase areas is foreseen. Equilibration is required in order to reduce the vertical incoherent tune shift during the rather long stacking time in the SIS100. Four to eight batches depending on the injection energy of the SIS18 beams can be transferred to the SIS100 consecutively and stacked longitudinally in a barrier bucket. In this manner a beam containing $1-2 \times$ $10^{12}$ particles can be generated in the SIS100. The design of the SIS100 dipole magnets will allow a field ramping speed of $4 \mathrm{~T} / \mathrm{s}$. Typical final energies for beam-matter interaction experiments would lie between $400 \mathrm{MeV} / \mathrm{u}$ to $2.7 \mathrm{GeV} / \mathrm{u}$ and a single bunch will be created after acceleration.

First a prebunching has to be achieved by means of a dynamic barrier bucket and then this compressed coasting beam will be captured in a harmonic rf potential operating at $h=2$. In order to avoid strong interactions with the impedance of the bunch compressor cavities, the rf gaps will be short-circuited by fast metaloxide-semiconductor field-effect transistor (MOSFET) switches during the whole acceleration process. Shortly before the beginning of the final bunch compression, the MOSFET switches will be opened. The compressor system will consist of 35 magnetic alloy loaded cavities operating at a nominal frequency of $465 \mathrm{kHz}$. A similar cavity is presently being developed for pulse compression of the SIS18 beam. The compressor system will be able to produce a maximum compression voltage of up to $1 \mathrm{MV}$ for the time of a quarter of a synchrotron period. The applied compression scheme, the so-called fast compression requires a fast jump in rf voltage to initiate a rotation of the longitudinal phase space. This leads to a very short pulse length of 20-90 ns, depending on the particle energy. The limit for compression is given by the maximum acceptable momentum spread of about $1 \%$. The generated short, high intensity bunch will be guided to the experimental area where the strong transverse focusing would be done either by a superconducting quadrupole final focusing system or by a plasma lens in front of the target. A schematic diagram of the accelerator chain for the beam-matter interaction experiments is shown in Fig. 1.

Experiments [1-5] and numerical simulations [7-9] have shown that intense heavy ion beams are an ideal tool for creating extended volumes of high-energydensity matter. However, diagnosing such samples is not

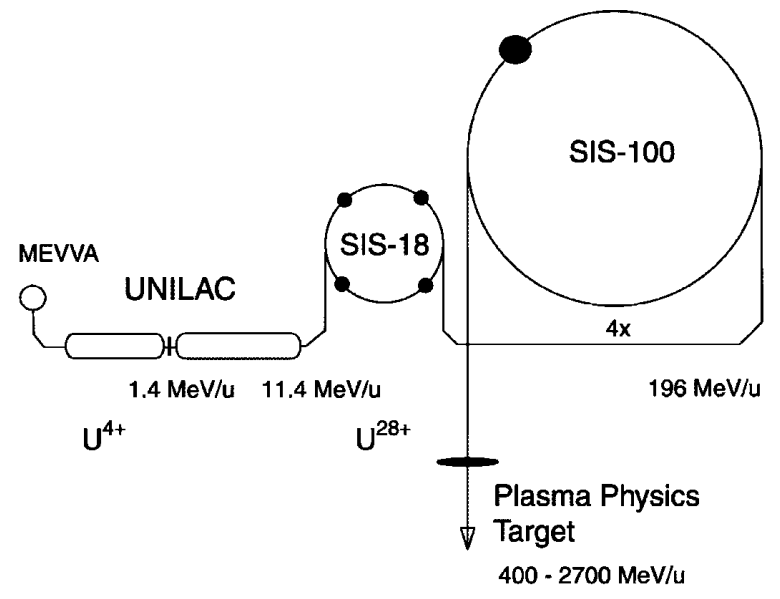

FIG. 1. A schematic diagram of the accelerator chain for beam-matter interaction experiments. 
an easy problem and one must develop new diagnostic tools to study such systems. A new method has been developed at the GSI [5] in which the same ion beam that heats the target has been used to diagnose the target by measuring the energy loss dynamics of the projectile ions in the target material. This method, of course, requires that the bunch length is large so that the ions that are delivered in the latter part of the pulse pass through the heated target which has already been subjected to hydrodynamic expansion/compression. We also know that for beam-matter heating it is advantageous to have the shortest possible pulse length, and the future SIS100 facility has been designed to include this important feature. This very useful method therefore cannot be employed to the experiments to be performed at the future SIS100 facility. To overcome this difficulty, it has already been proposed for the SIS18 [10] to accelerate two beams simultaneously: a main high intensity bunched beam (short pulse length) that is composed of a heavy element and a very low intensity unbunched beam (with a pulse length of the order of $1 \mu \mathrm{s}$ ) which is made of a lighter element. A similar scheme is foreseen for the SIS100 facility. The main beam will deliver its energy in a few tens of ns that will heat the target, leading to the onset of hydrodynamics. The second beam will be available for a much longer time, and a study of the energy loss dynamics of this beam in the target material will provide time resolved information about the physical state of the target. Another possibility is to consider that the driver and the diagnostic beam are made of the same species (for example, U). In this case the accelerator has to deliver a single bunch with an appropriate time structure. The existing GSI accelerator has the possibilities to provide beams for the HED experiments using both the above mentioned schemes, as described below.

\section{A. Two beams of different ion species}

Two beams of different ions can be accelerated in a heavy ion synchrotron SIS18 if the magnetic rigidity is equal over the whole acceleration cycle. Furthermore, the difference in revolution frequency must be big enough that the individual beams can be handled by the two separate rf acceleration systems without interference [10]. The two ion beams may be produced in parallel by two different ion sources. Two high voltage terminals as required are available at GSI, for example, the uranium beam may be generated in the north terminal by a MEVVA source, while the argon beam is generated in the south terminal by a PIG (penning) source. For the existing SIS18 facility it has been proposed to accelerate two beams in the UNILAC up to slightly different energies for injection in SIS18. The injection energy can be optimized by choosing an appropriate charge over mass ratio of the ions and has to fit into the possible final energy range of UNILAC. A possible combination is
$\mathrm{Ar}^{5+}$ and $\mathrm{U}^{28+}$ ion beams with injection energies of $12.8 \mathrm{MeV} / \mathrm{u}$ and $11.4 \mathrm{MeV} / \mathrm{u}$, respectively.

The sophisticated GSI accelerator control system enables a fast repetitive multiturn injection. This is applied at the operation with the so-called multiturn injection process, where the SIS18 electron cooler is used to gain beam intensity and quality.

After acceleration and debunching, the preparation of the $U$ beam for optimal target heating can be performed using both rf acceleration systems and eventually a third rf system for compression. Finally, the compressed U beam with a pulse length of $50 \mathrm{~ns}$ and the coasting Ar beam of a pulse duration of $1.3 \mu$ s can be fast extracted out of the synchrotron and delivered to the experimental area. An appropriate time structure would be such that the short U pulse is leading the long Ar pulse. Such a time structure can be achieved by an appropriate timing of the extraction kicker.

\section{B. Single beam of same ion species but with a specific time structure}

A high intensity heavy ion beam (for example, $\mathrm{U}^{28+}$ ) is accelerated in SIS18 up to the final energy. An appropriate time structure of a beam for plasma generation and simultaneous diagnostics should have a leading peak for heating, followed by a low level tail of sufficient length for diagnostics. Such a time structure can be achieved by a nonlinear bunch compression. Normally, fast bunch compression starts from a sufficiently prebunched beam. If the beam is not adequately prebunched or not prebunched at all, nonlinear $\mathrm{rf}$ forces distort its initially elliptical longitudinal phase space area. After a $90^{\circ}$ phase space rotation, the final distribution in longitudinal phase space becomes strongly $s$ shaped, as shown in Fig. 2. The particle distribution in real space consists of a narrow peak in the center, followed by a low intensity tail. Most extreme is the compression starting at harmonic number

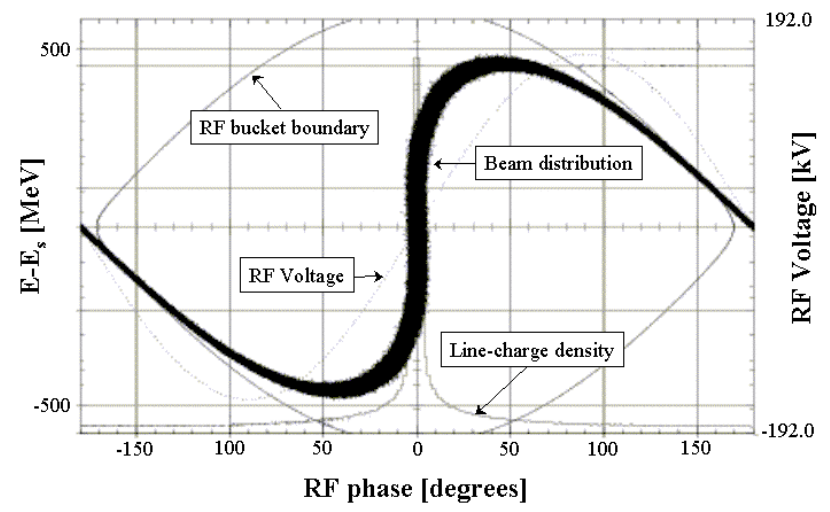

FIG. 2. (Color) The figure shows the beam properties after nonlinear compression. Plotted are the $s$-shaped phase space area, the rf bucket area (solid line), the harmonic rf voltage (dotted line), and the particle distribution in the longitudinal direction. 


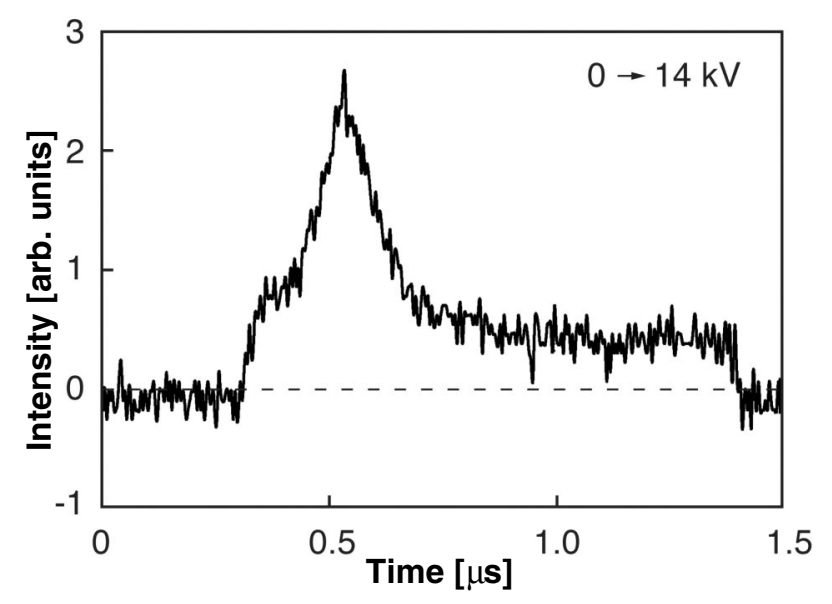

FIG. 3. Beam profile after nonlinear compression by means of an rf voltage jump from 0 to $14 \mathrm{kV}$ and an appropriate extraction kicker timing.

one from a coasting beam (without prebunching). Figure 2 shows the resulting beam properties obtained by using the ESME computer code which calculates the evolution of a distribution of particles in energy and azimuth as it is acted upon by a radio frequency system of a synchrotron or a storage ring. The figure shows the longitudinal phase space area energy deviation from a reference particle energy, $E-E_{s}$, vs rf phase space after compression of a $\mathrm{U}^{28+}$ in SIS18 with a compression voltage of $160 \mathrm{kV}$ starting from a coasting beam. Furthermore, the particle distribution in real space (line charge density), the rf voltage, and the rf bucket as a function of the phase angle are plotted. If such a beam is extracted with an appropriate timing of the extraction kicker, a time structure as shown in Fig. 3 can be obtained [11]. The time structure shown was measured close to the target position by a fast beam transformer. Plotted is the longitudinal profile of an Ar beam after nonlinear compression at $300 \mathrm{MeV} / \mathrm{u}$. The compression was achieved by applying an rf-voltage jump of $14 \mathrm{kV}$ with the existing acceleration cavities of SIS18. This time structure obtained during machine experiments would already be suitable for target heating and subsequent diagnostic. Within some limits the time structure after compression can be matched to the target requirements by choosing other sets of rf parameters.

This scheme will also be considered for the SIS100 scenario.

\section{SIMULATION RESULTS}

In this section we present the numerical simulation results. These results have been obtained by employing a two-dimensional hydrodynamic simulation model, BIG-2 [6].

\section{A. Creation of different phases of matter by heavy ion heating}

In this study we considered the beam-target geometry shown in Fig. 4(a). The target is a solid lead cylinder
SubRange Heavy Ion Driven Targets

(a) Solid Cylinder

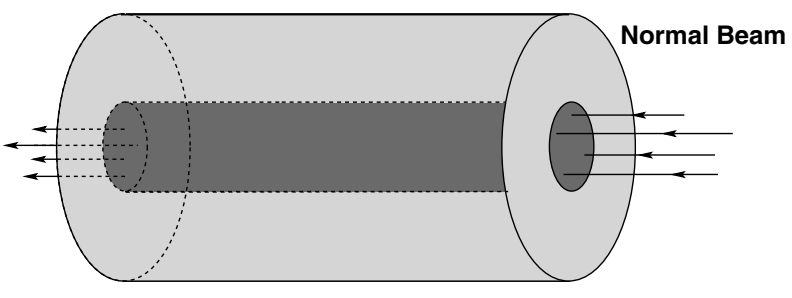

(b) Solid Cylinder

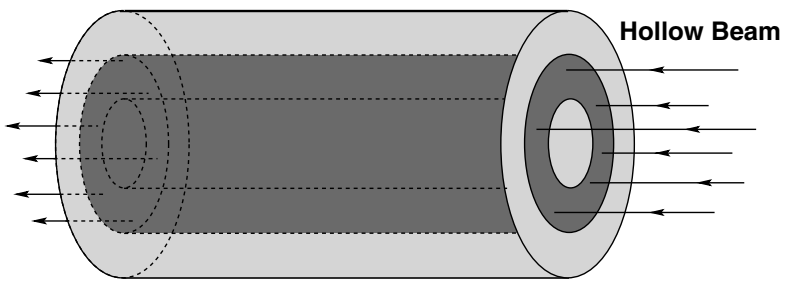

(c) Hollow Cylinder

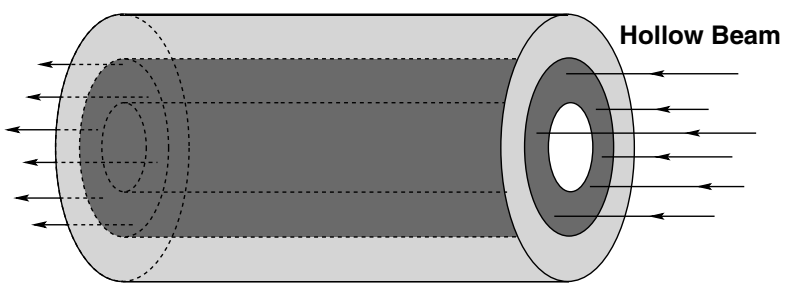

FIG. 4. Beam-target geometry for three proposed experiments: (a) a solid cylinder irradiated with a beam having a circular focal spot, (b) a solid cylinder irradiated with a hollow beam, and (c) a hollow cylinder irradiated with a hollow beam.

which has a length, $L=1.0 \mathrm{~cm}$, and a radius, $r_{o}=$ $2.5 \mathrm{~mm}$. The left face of this cylinder is irradiated with the SIS100 beam. For this particular configuration we consider a uranium beam with a particle energy of $2.7 \mathrm{GeV} / \mathrm{u}$, having an intensity, $N=1.5 \times 10^{12}$ ions which are delivered in a single bunch having a duration, $\tau=20 \mathrm{~ns}$. We also assume that the beam radius (FWHM of the Gaussian distribution) is $1.0 \mathrm{~mm}$. The range of the $2.7 \mathrm{GeV} / \mathrm{u}$ uranium ions in solid cold lead is $5.95 \mathrm{~cm}$ [12], which is much larger than the length of the target. The projectile ions will therefore deposit a fraction of their energy very uniformly along their trajectory in the target and will emerge from the opposite face of the cylinder with a reduced energy. We note that in these calculations we use the multiphase, semiempirical equation-of-state (EOS) model for lead developed at Chernogolovka [13,14].

In Fig. 5 we plot the specific energy deposited by the beam in the target on a length-radius diagram at $t=$ $20 \mathrm{~ns}$, a time when the beam has just delivered its total energy. It is seen that the specific energy deposition has a 


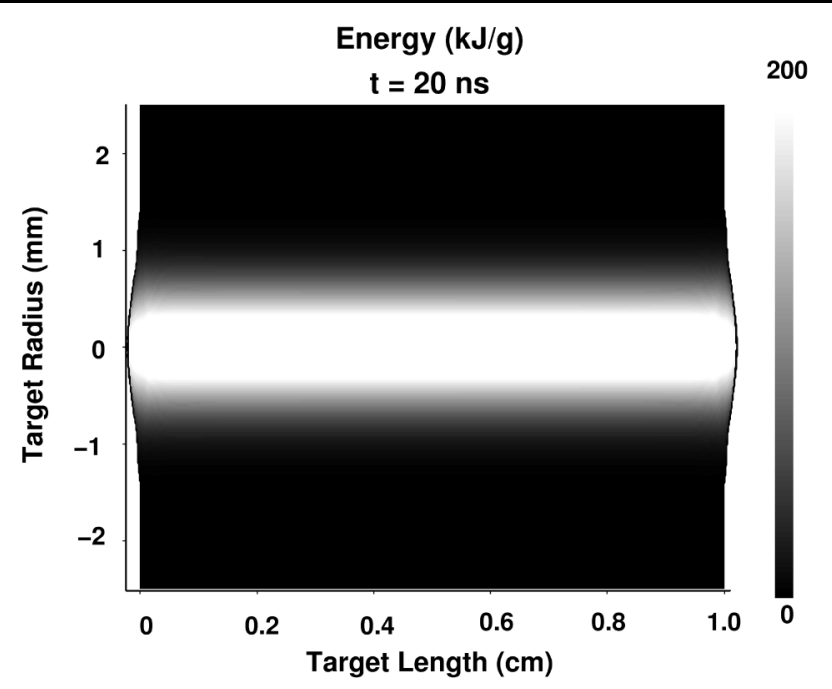

FIG. 5. Specific energy deposition on a length-radius plane employing the beam-target geometry shown in Fig. 4(a), using a uranium beam having $1.5 \times 10^{12}$ particle of $2.7 \mathrm{GeV} / \mathrm{u}$ uranium, focal spot radius (full width at half maximum) $=$ $1.0 \mathrm{~mm}$, pulse duration, $\tau=20 \mathrm{~ns}$. The target is a cylinder made of solid lead with a length $L=1.0 \mathrm{~cm}$ and radius $=$ $2.5 \mathrm{~mm}$.

maximum value of $200 \mathrm{~kJ} / \mathrm{g}$ at the target axis where the maxima of the Gaussian distribution lie. This energy profile gives rise to a corresponding temperature profile which has a maximum value of the order of $18 \mathrm{eV}$. The pressure in this heated region also increases substantially as shown in Fig. 6. It is seen that a maximum pressure of 9.2 Mbar is generated at the axis.

The high pressure in the absorption region generates a strong shock wave that travels outward, along the radial direction. Also the material from the heated region expands along the axial direction. In Figs. 7-9 we plot the target density on a length-radius diagram at three differ-

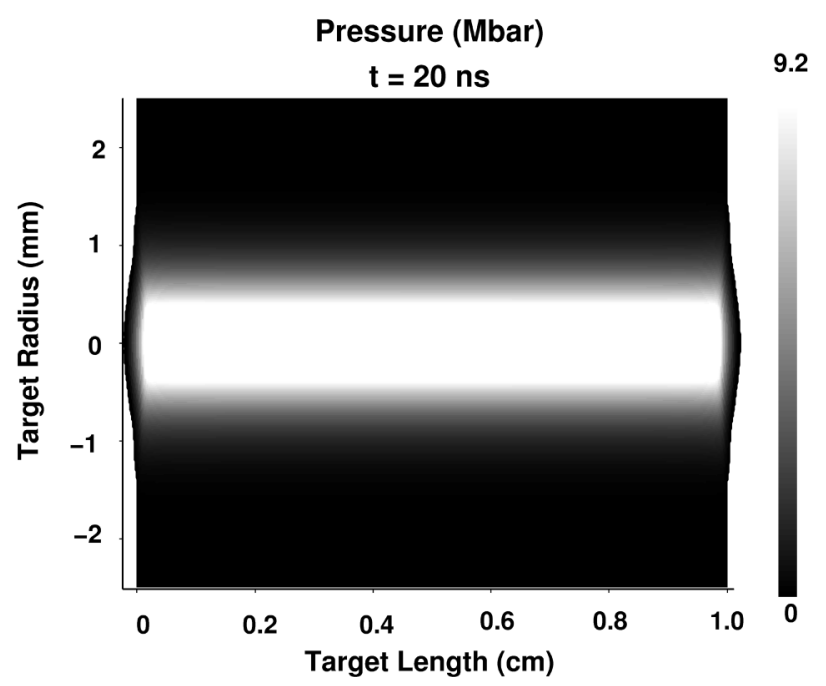

FIG. 6. Pressure corresponding to the case shown in Fig. 5.

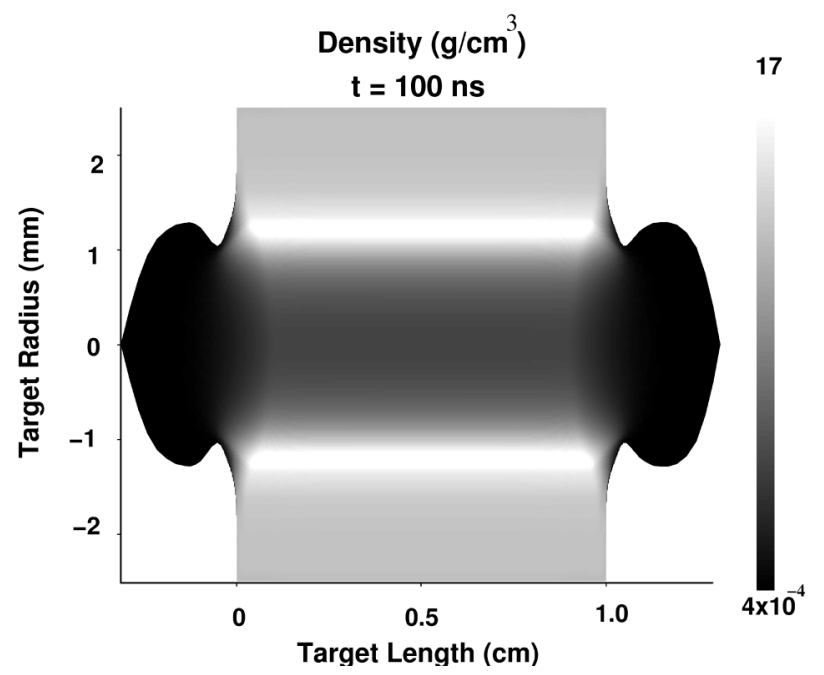

FIG. 7. Density at $t=100 \mathrm{~ns}$ corresponding to the case shown in Fig. 5.

ent times, namely, $t=100 \mathrm{~ns}, 160 \mathrm{~ns}$, and $200 \mathrm{~ns}$, respectively.

It is seen from Fig. 7 that a shock wave has developed and the density in the shock region is $17 \mathrm{~g} / \mathrm{cm}^{3}$. At the same time, there is a plume of target material in the axial direction on both sides of the cylinder. Figure 8 shows that the shock wave has traveled a larger distance away from the axis and the density has increased in the shock region. This is because there is a characteristic time for the shock to achieve its maximum strength. Also it is seen from this figure that the material expansion along the cylinder length has increased significantly.

Figure 9 shows that the shock density has now been reduced slightly to a value of $20.9 \mathrm{~g} / \mathrm{cm}^{3}$, which is due to the cylindrical geometry.

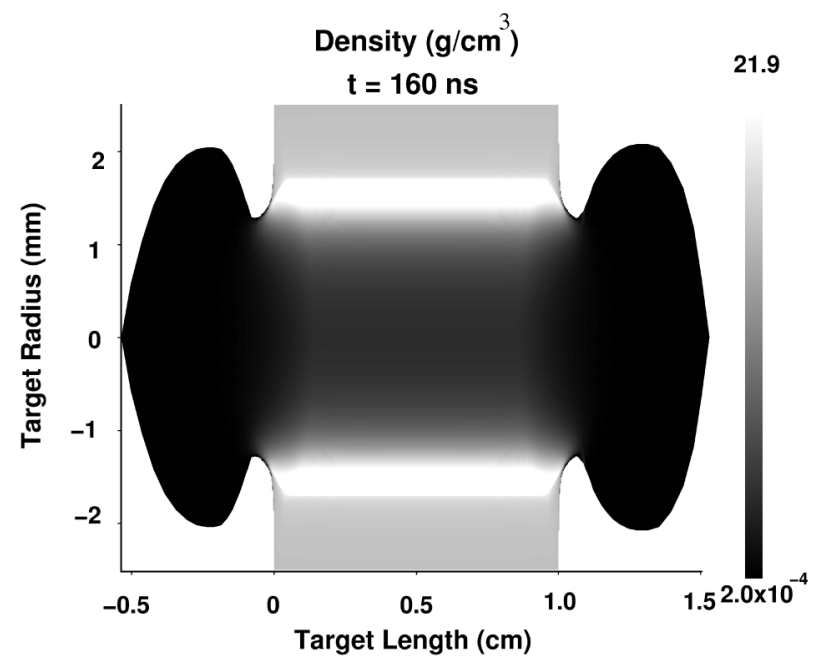

FIG. 8. Density at $t=160 \mathrm{~ns}$ corresponding to the case shown in Fig. 5. 


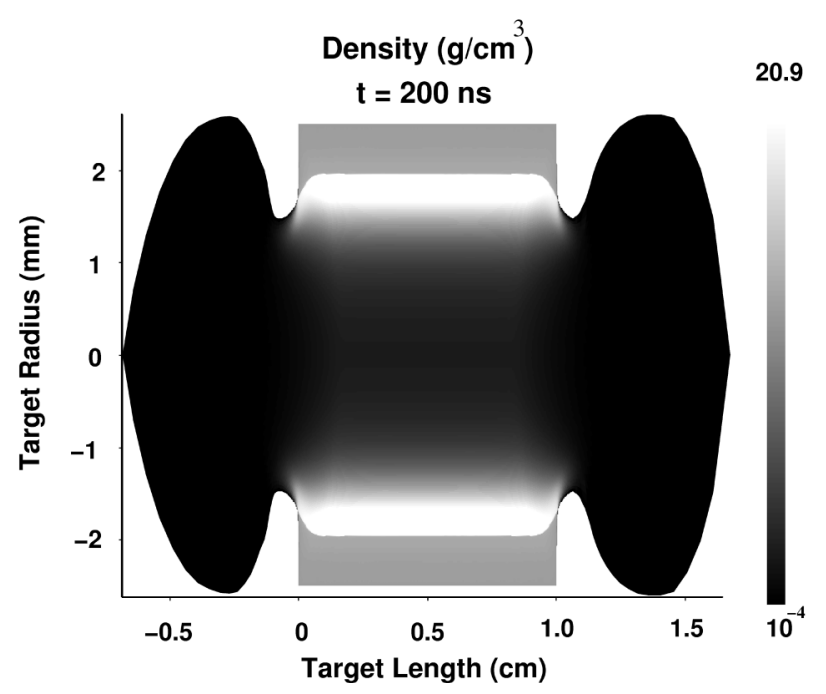

FIG. 9. Density at $t=200 \mathrm{~ns}$ corresponding to the case shown in Fig. 5.

The target temperature corresponding to the above density figures is plotted in Figs. 10-12 respectively. It is seen that the temperature decreases with time due to the hydrodynamic response of the target. The maximum temperature values at the cylinder axis at 100, 160, and $200 \mathrm{~ns}$ are $12.5,11$, and $10.6 \mathrm{eV}$, respectively.

The change of temperature and density due to the beam deposition and hydrodynamic effects leads to phase changes in the target material. In Figs. 13-15 we plot the material phase state corresponding to the density and temperature distributions (at $t=100 \mathrm{~ns}, 160 \mathrm{~ns}$, and $200 \mathrm{~ns}$, respectively). Figure 13 shows that at $t=$ $100 \mathrm{~ns}$, the inner part of the target including the shock compressed region is in a liquid phase [denoted by a number (3)] while the unshocked region is still in a solid phase [denoted by number (1)]. The expanded region at

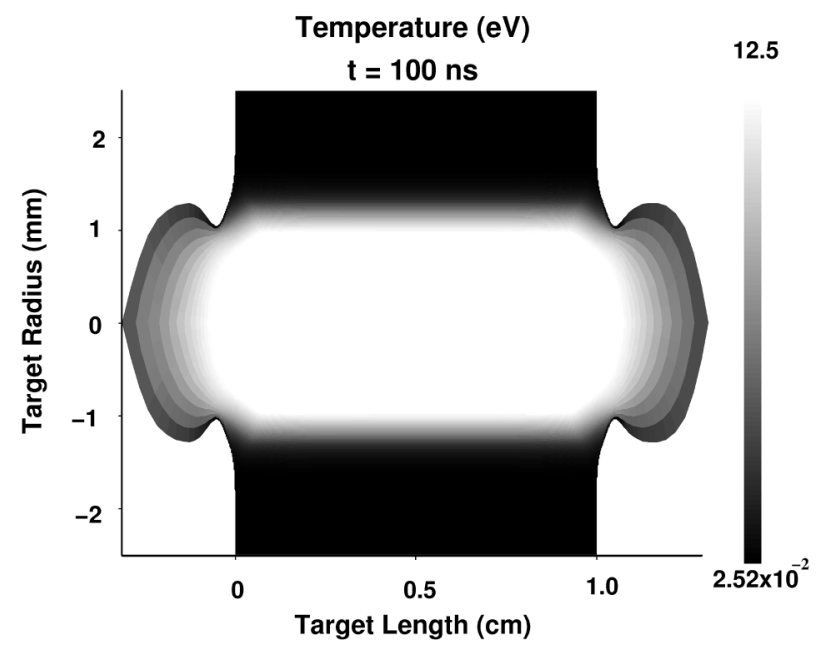

FIG. 10. Temperature at $t=100 \mathrm{~ns}$ corresponding to the case shown in Fig. 5.

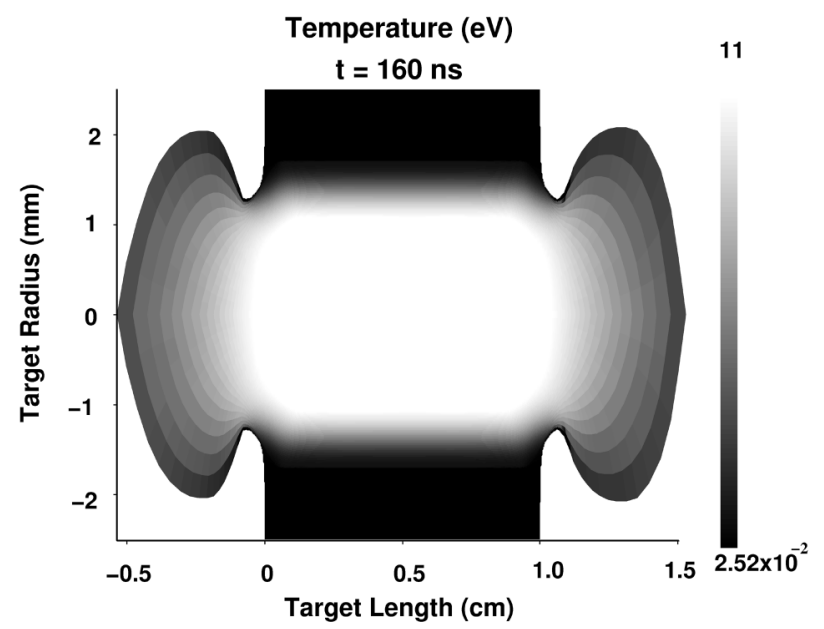

FIG. 11. Temperature at $t=160 \mathrm{~ns}$ corresponding to the case shown in Fig. 5.

both faces of the cylinder, on the other hand, is in a plasma state [denoted by a number (5)]. This EOS assumes that the material will be a gas if the temperature becomes higher than the critical point temperature and the density is below the density at the critical point. The temperature in the present case is high enough so that the gas will be weakly ionized.

Figure 14 shows that at $t=160 \mathrm{~ns}$, not only do we have a plasma in the expanded region, but we also have a plasma created in a small cylindrical region around the target axis. Figure 15 shows that at $t=200 \mathrm{~ns}$ the plasma region has significantly expanded.

Since the temperature in the plasma region is low (of the order of a few $\mathrm{eV}$ ) while the density in the middle of the target is high enough (1/5-1/10 of solid density), one would be able to generate nonideal plasmas in the laboratory using this device. An experimental study of the EOS of such nonideal plasmas and studies of energy loss

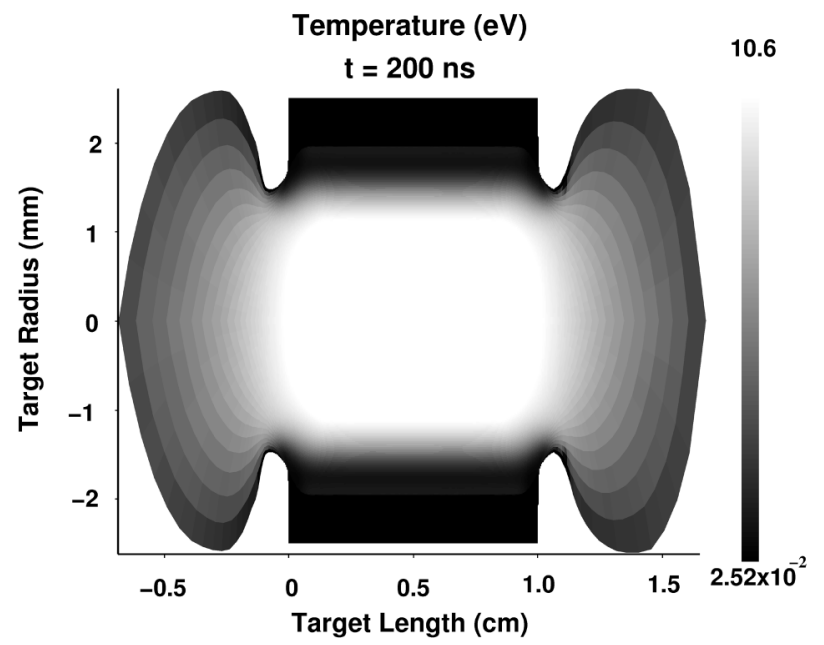

FIG. 12. Temperature at $t=200 \mathrm{~ns}$ corresponding to the case shown in Fig. 5. 


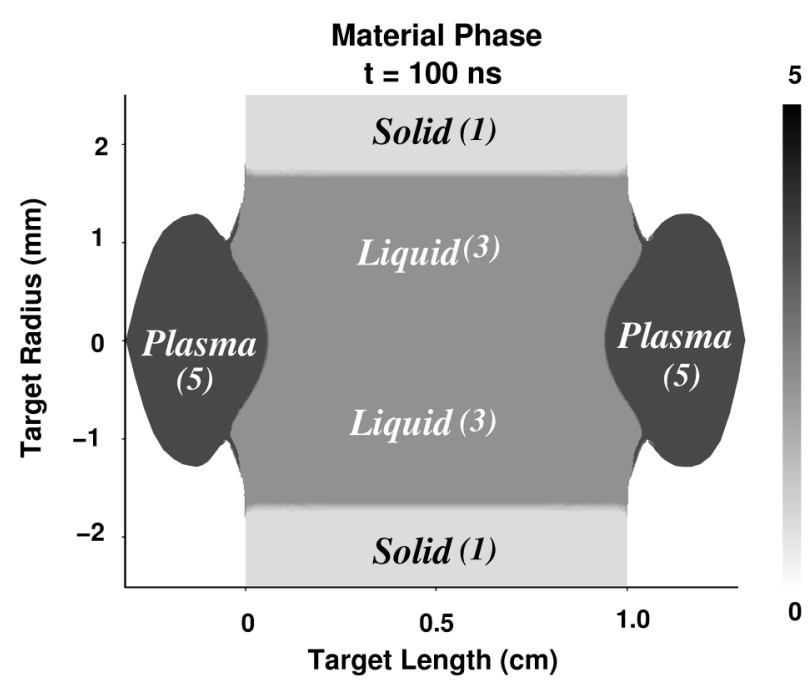

FIG. 13. Matter state at $t=100 \mathrm{~ns}$ corresponding to the case shown in Fig. 5.

of ions in such samples will be a valuable contribution to the physics of these complicated systems which occur frequently in nature. Use of an intense beam to study the EOS properties of matter has a number of advantages over other techniques used in this field. These include a diamond anvil cell, gas guns, high power lasers, and high power explosive devices [15-23].

In a previous paper [24] we have shown that an intense ion beam is an ideal tool to heat matter isochorically. If one allows this heated matter to expand along the release isentrope, it will pass through different states of matter, including nonideal plasmas. This way one can easily access those parts of the phase diagrams which have not been previously explored. In the type of experimental design discussed in this section one can study the properties of the material expanding along the axial direction of

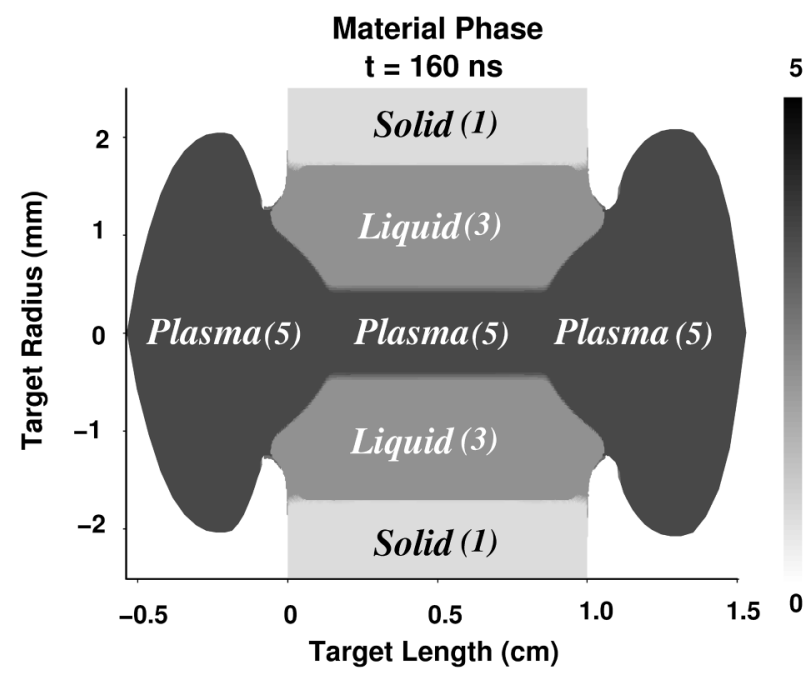

FIG. 14. Matter state at $t=160 \mathrm{~ns}$ corresponding to the case shown in Fig. 5.

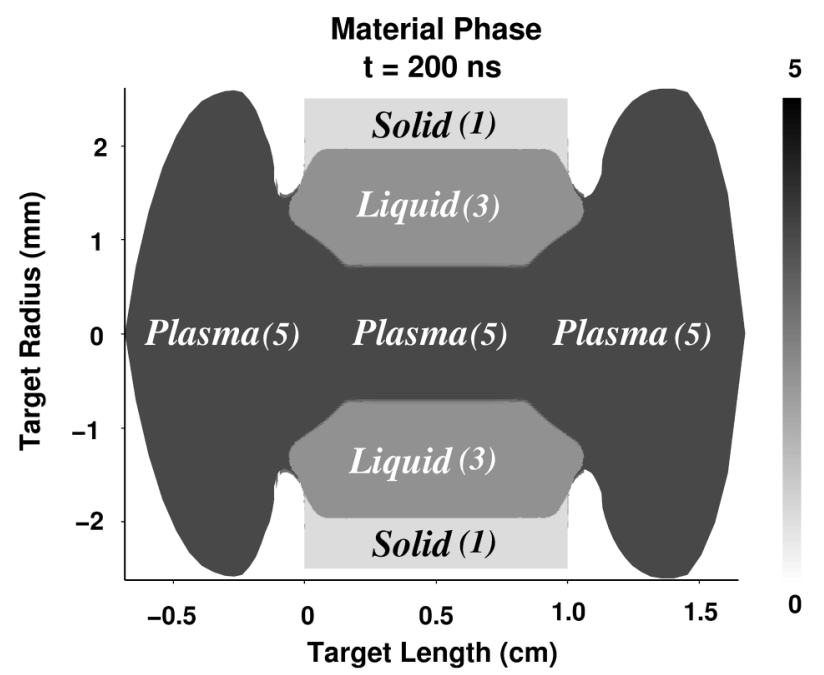

FIG. 15. Matter state at $t=200 \mathrm{~ns}$ corresponding to the case shown in Fig. 5.

the target. Experiments of this type are also being planned at the GSI for the future.

\section{B. A comparison between semiempirical EOS and the SESAME EOS data}

Considering the same beam-target geometry and the same beam and target parameters as in the previous subsection, we have also carried out calculations using the SESAME EOS data [25]. In this subsection we present a comparison between the results obtained using these two models.

In Fig. 16 we plot the specific energy deposition vs target radius, at $L=0.5$ (middle of the cylinder axis). The corresponding temperature and pressure profiles are presented in Figs. 17 and 18, respectively. Figure 17 shows that the maximum temperature using the semiempirical EOS model is about $18 \mathrm{eV}$, while the SESAME data lead to a higher temperature of $21.5 \mathrm{eV}$, a difference of about $16 \%$.

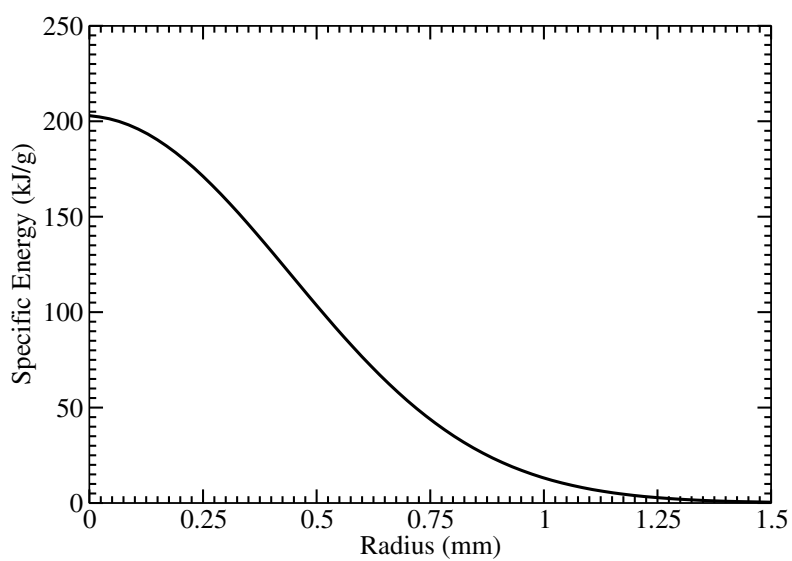

FIG. 16. Specific energy deposition vs radius at $t=20 \mathrm{~ns}$ corresponding to the case shown in Fig. 5. 


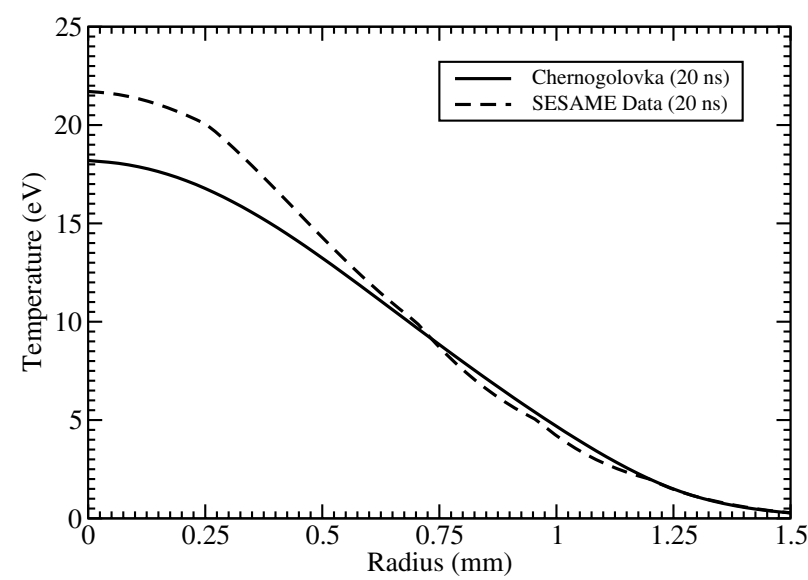

FIG. 17. Temperature vs radius at $t=20 \mathrm{ns,} \mathrm{using} \mathrm{the} \mathrm{beam}$ and target parameters shown in Fig. 5, but using two different equation-of-state models, namely, SESAME and a semiempirical model.

This is because the former model provides a colder liquid in comparison to SESAME as it takes into account the effect of melting and of thermal electron contribution from zero temperature. In addition, the semiempirical model includes the contribution of the thermal electrons from the degenerated electron gas in crystal to ionized metal at high temperatures.

The pressure, on the other hand, shows a behavior opposite to the temperature as one achieves 9.4 Mbar using the semiempirical model and 8.4 Mbar using the SESAME data which is a difference of about $8 \%$. The matter in the target corresponds to quasi-ideal weakly degenerated electron gas with a high concentration of energy $(200 \mathrm{~kJ} / \mathrm{g})$. The difference between the results in the two calculations is due to the fact that the SESAME uses a Thomas-Fermi model, which produces lower thermal pressure compared to the semiempirical model. It is

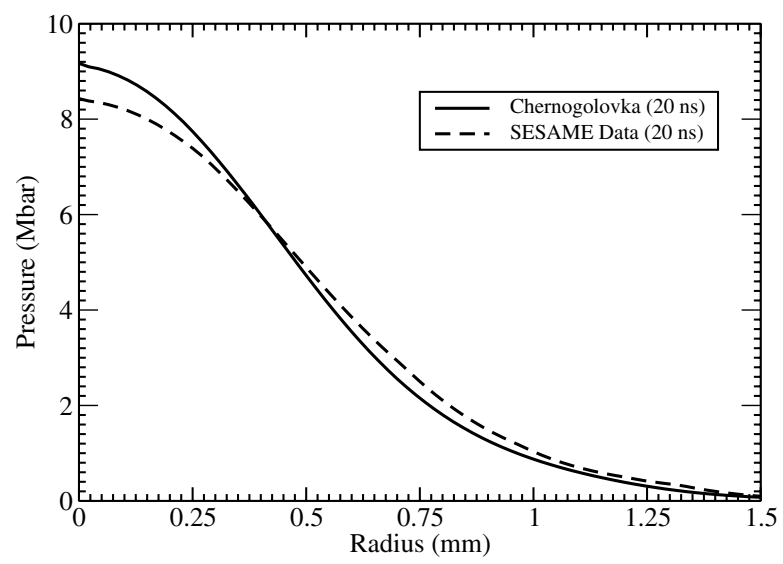

FIG. 18. Pressure vs radius at $t=20 \mathrm{~ns}$, using the beam and target parameters shown in Fig. 5, but using two different equation-of-state models, namely, SESAME and a semiempirical model.

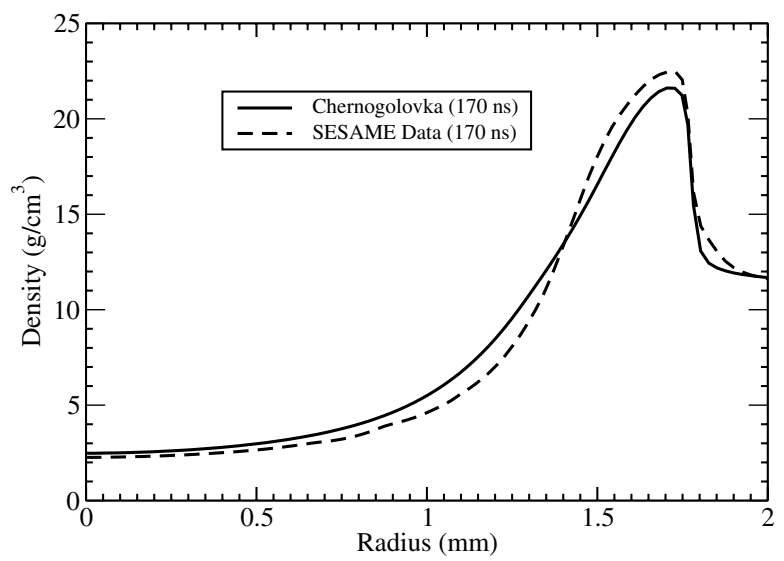

FIG. 19. Density vs radius at $t=170 \mathrm{~ns}$ for the calculations presented in Fig. 17.

to be noted that these profiles are plotted at $t=20 \mathrm{~ns}$ when the beam has just delivered its total energy and the target has still solid density because there has been no hydrodynamic motion yet.

The high pressure in the absorption region drives a strong shock wave along the radius in the outward direction as shown in Fig. 19 where we plot the density vs radius at $t=170 \mathrm{~ns}$. It is seen that the density evolution in the case of the above two EOS models is comparable.

These calculations show that the SESAME EOS data for lead is in reasonably good agreement with the multiphase semiempirical EOS model. This is the case for most of the metals.

\section{Target calculations using an elliptic focal spot}

The calculations presented in the previous two subsections assume a circular focal spot whereas in many cases of practical importance it may be preferable to consider an elliptic focal spot, for example, in the case of fragment separator experiments [26]. We therefore have carried out calculations using an elliptic beam and the results are presented below.

We assume that the focal spot can be represented by a two-dimensional Gaussian distribution with a standard deviation $\sigma_{x}$ along the $x$ direction and $\sigma_{y}$ along the $y$ direction. Also $\sigma_{x}=0.3 \mathrm{~mm}$ and $\sigma_{y}=0.6 \mathrm{~mm}$ so that the FWHM in the $x$ direction is $0.7 \mathrm{~mm}$ and in the $y$ direction is $1.4 \mathrm{~mm}$. The target parameters and the rest of the beam parameters are the same as used in the previous two subsections.

In Fig. 20 we plot the specific energy deposition vs the target radius along the $x$ direction and the $y$ direction, respectively, at $t=20 \mathrm{~ns}$ (when the pulse has just delivered its total energy). The corresponding pressure profiles are given in Fig. 21. It is seen from Fig. 21 that the pressure gradient along the $x$ direction is much steeper than that in the $y$ direction. As a result, a stronger shock wave is generated along the $x$ direction compared to the $y$ 


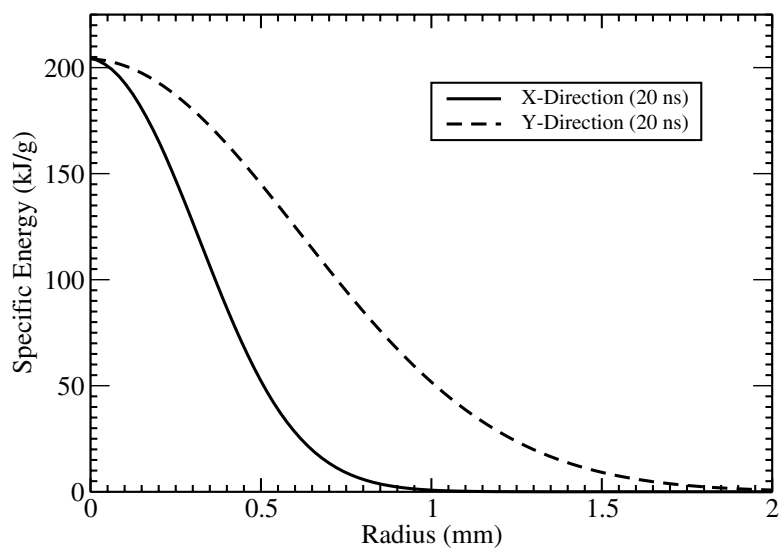

FIG. 20. Specific energy deposition vs radius ( $x$ and $y$ directions) at $t=20 \mathrm{~ns}$, using an elliptic focal spot, $\sigma_{x}=0.3 \mathrm{~mm}$ and $\sigma_{y}=0.6 \mathrm{~mm}$. The rest of the beam parameters and the target parameters are the same as in the case of Fig. 5 .

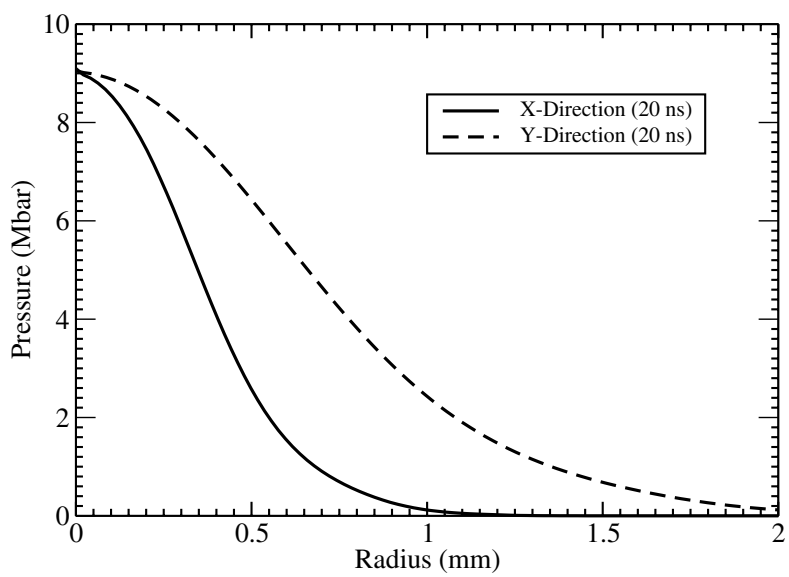

FIG. 21. Pressure vs radius corresponding to the case shown in Fig. 20.

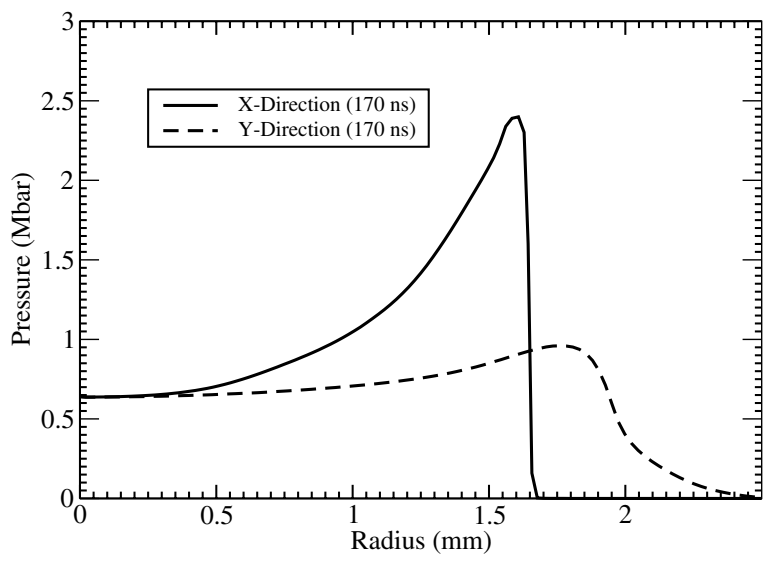

FIG. 22. Same as in Fig. 21, but at $t=170 \mathrm{~ns}$.

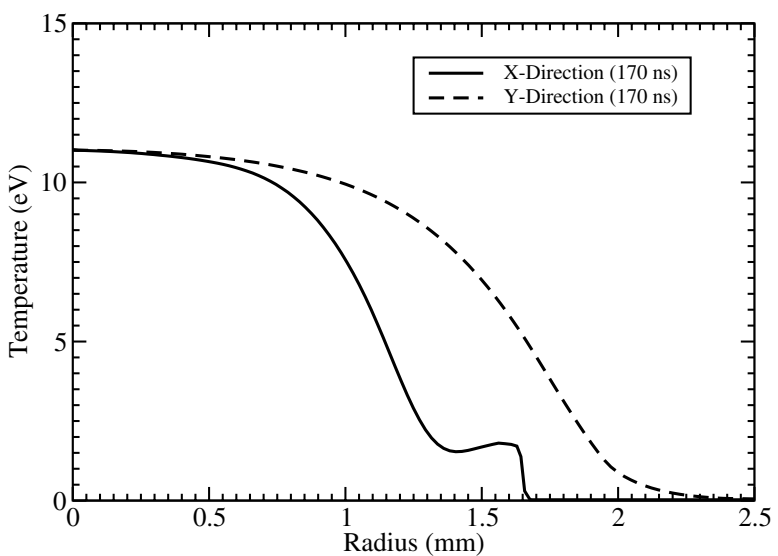

FIG. 23. Temperature corresponding to the case presented in Fig. 22.

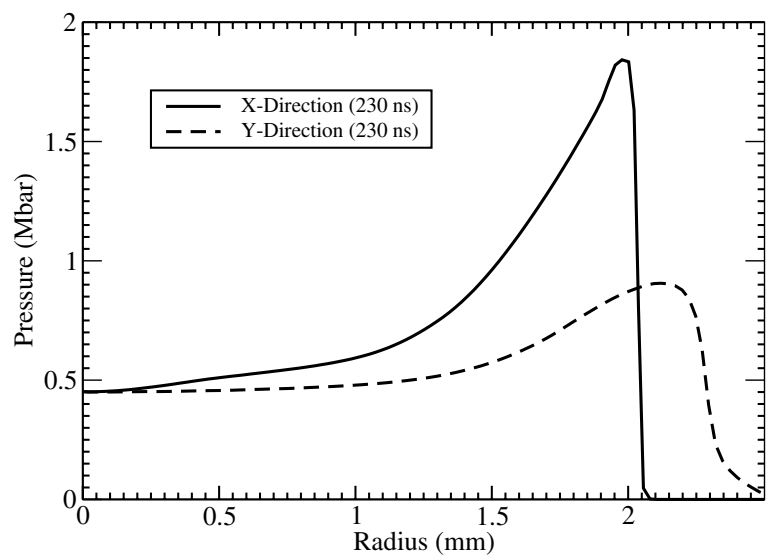

FIG. 24. Same as in Fig. 21, but at $t=230 \mathrm{~ns}$.

direction. Moreover, the development of the shock wave takes place earlier along the $x$ direction as compared to the $y$ direction. This is clearly seen from Fig. 22 where we plot the pressure vs the $x$ and the $y$ directions at $t=$ 170 ns. In Fig. 23 we plot the corresponding temperature profiles at $t=170 \mathrm{~ns}$. It is interesting to see that the temperature profile along the $x$ direction ends in a welldefined step shape (at $r=1.65 \mathrm{~mm}$ ) which lies in the shock compressed region. On the other hand, the temperature profile along the $y$ direction decreases monotonically and smoothly because along this direction there has been no strong shock wave development.

Figures 24 and 25 show the pressure and the temperature profiles at a later time, $t=230 \mathrm{~ns}$. It is seen that the behavior of the curves remains the same as in Figs. 22 and 23 , except that the pressure and the temperature values have decreased.

\section{Target calculations using two beams}

We have already described how one may accelerate two beams in the accelerator at the same time and the target 


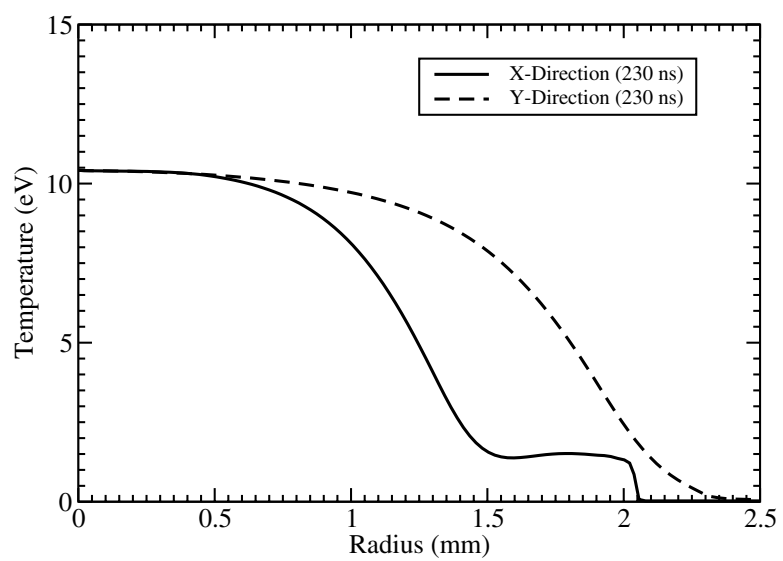

FIG. 25. Same as in Fig. 23, but at $t=230 \mathrm{~ns}$.

may be irradiated by these two beams simultaneously. In this subsection we present simulations of different target designs that are irradiated by two beams simultaneously. We assume that the main beam that heats the target is the SIS100 beam which is made of $\mathrm{U}^{28+}$ particles. In these calculations we assume a particle energy of $1 \mathrm{GeV} / \mathrm{u}$, a total number of $10^{12}$ ions that are delivered in a single bunch having a length of $50 \mathrm{~ns}$. For the diagnostic beam we use two options. First we consider an $\mathrm{Ar}^{5+}$ beam with a particle energy of $1.095 \mathrm{GeV} / \mathrm{u}$ so that the two species of ions have the same magnetic rigidity. The intensity of the diagnostic beam is $10^{6}$ particles which are delivered in a $1 \mu$ s pulse. The second option is to use the diagnostic beam made of the same species and energy as the main beam, but with a much lower intensity and a much longer pulse duration. We therefore consider a diagnostic beam of $1 \mathrm{GeV} / \mathrm{u}$ uranium with an intensity of $10^{6}$ and a pulse length of $1 \mu \mathrm{s}$.

First we consider the beam-target arrangement shown in Fig. 4(a). The target is a solid lead cylinder which is $0.5 \mathrm{~cm}$ long and the main beam as well as the diagnostic beam has a circular focal spot. The range of $1 \mathrm{GeV} / \mathrm{u}$ uranium ions in solid cold lead is $1.55 \mathrm{~cm}$ and therefore the projectile ions deposit a fraction of their energy in the target and emerge from the opposite face of the cylinder.

The main beam delivers its energy in $50 \mathrm{~ns}$ and heats the target very strongly. The pressure in the heated region increases substantially driving an outgoing shock wave along the radial direction. As a result of this radial shock wave, the linear density along the cylinder axis decreases with time and therefore the particles of the diagnostic beam lose less and less energy in the target. This leads to an increase in the fraction of energy of these particles that escape the target. This energy curve therefore can provide time resolved information about the target density. Experimental measurement of this type of energy curve has already been done in a number of interesting experiments at the GSI using the existing accelerator facility SIS18 [6].
In Fig. 26 we plot the line density along the cylinder axis vs time and the fraction of the energy of the ions of the diagnostic beam escaping along the axis. It is seen that the line density decreases from an initial value of $5.67 \mathrm{~g} / \mathrm{cm}^{2}$ to about $1 \mathrm{~g} / \mathrm{cm}^{2}$ and consequently for the uranium diagnostic beam, the particle energy escaping from the target increases from $75 \%$ to $96 \%$ of the initial energy over a period of $300 \mathrm{~ns}$. The Ar ions have a much longer range than the uranium ions and therefore they lose less energy in the target. It is seen from Fig. 26 that initially about $95 \%$ ion energy escapes the target in this case while after $300 \mathrm{~ns}$ almost $100 \%$ energy is carried out of the target. Such a small energy difference is not easily measurable and therefore one should use a heavier species for the diagnostic beam in this type of design.

Next we consider the beam-target geometry shown in Fig. 4(b). The target is a solid lead cylinder that is $6.0 \mathrm{~mm}$ long and the main beam, the SIS100 beam, has an annular or a ring shaped focal spot. The inner radius of the ring is $R_{1}=0.5 \mathrm{~mm}$, while the outer radius is $R_{2}=1.5 \mathrm{~mm}$. The target radius is $3.0 \mathrm{~mm}$. The beam deposits energy in the target and a ring shaped hot, high-pressure zone is created in the target in the region between $R_{1}$ and $R_{2}$. This high pressure generates a strong shock wave that moves inwards and outwards along the radial direction. The density increases substantially when the shock wave converges at the axis which leads to a significant increase in the line density along the axis. We again consider two different cases for the diagnostic beam, $1 \mathrm{GeV} / \mathrm{u}$ uranium and $1.095 \mathrm{GeV} / \mathrm{u}$ argon. In Fig. 27 we plot the same variables as in Fig. 26. In the beginning the line density

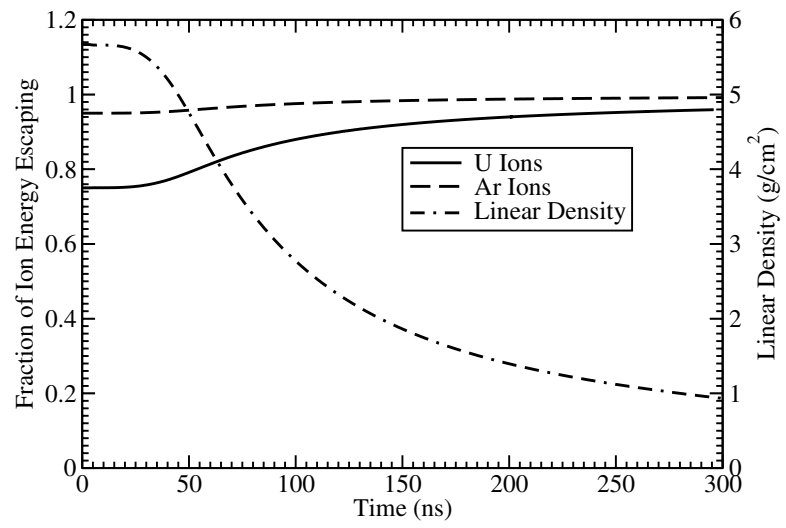

FIG. 26. Line density (linear density), fraction of energy of the ions of the diagnostic beam escaping the target vs time, employing the beam-target geometry shown in Fig. 4(a), using a main uranium beam having $10^{12}$ particles of $1.0 \mathrm{GeV} / \mathrm{u}$ uranium, focal spot radius (full width at half maximum) $=$ $1.0 \mathrm{~mm}$, pulse duration, $\tau=50 \mathrm{~ns}$. Two different diagnostic beams are considered, a uranium $1 \mathrm{GeV} / \mathrm{u}$ uranium beam with an intensity of $10^{6}$ particles and a $\tau=1 \mu \mathrm{s}$ and an argon beam with a particle energy of $1.095 \mathrm{GeV} / \mathrm{u}$, an intensity of $10^{6}$ and a $\tau=1 \mu \mathrm{s}$. The target is a cylinder made of solid lead with a length $L=5.0 \mathrm{~mm}$ and radius $=2.5 \mathrm{~mm}$. 


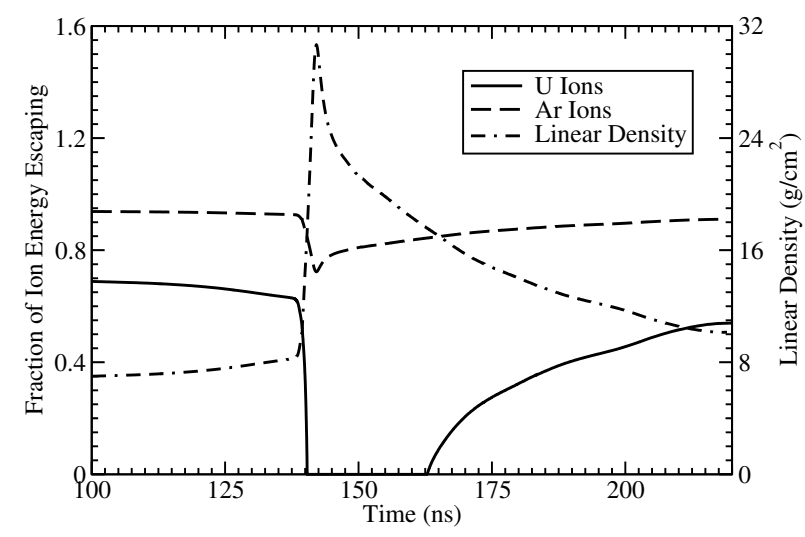

FIG. 27. Same as in Fig. 26, but using the beam-target geometry shown in Fig. 4(b).

along the cylinder axis is $6.8 \mathrm{~g} / \mathrm{cm}^{2}$, and $68 \%$ and $94 \%$ of the initial energies of the uranium and argon diagnostic beam ions, respectively, escape the target. It is seen that at $t=140 \mathrm{~ns}$, the line density increases suddenly to a value of about $30 \mathrm{~g} / \mathrm{cm}^{2}$ because at this time the shock wave arrives at the axis. The range of the $1 \mathrm{GeV} / \mathrm{u}$ uranium ions in solid cold lead is $1.55 \mathrm{~cm}\left(17.57 \mathrm{~g} / \mathrm{cm}^{2}\right)$ and therefore the particles of the uranium diagnostic beam are completely stopped in the target, as shown by the corresponding energy curve. As the shock wave is reflected at the axis, the line density starts to decrease along the axis and at $t=155 \mathrm{~ns}$ it becomes less than $17.57 \mathrm{~g} / \mathrm{cm}^{2}$ and the ions start to reemerge from the target.

In case of the argon ions, initially $94 \%$ of the initial ion energy escapes the target but at the time of maximum compression at the axis, the fraction of energy escaping the target decreases to about $75 \%$.

Finally, we present the results obtained using the geometry shown in Fig. 4(c). The target in this case is a hollow lead cylinder which is $6.0 \mathrm{~mm}$ long and is irradiated with a hollow beam. The inner cylinder radius, $R_{i}$, and the inner radius of the beam focal ring, $R_{1}$, are $0.5 \mathrm{~mm}$ each. The outer focal spot radius is $R_{2}=$ $1.5 \mathrm{~mm}$ and the outer cylinder radius is $R_{o}$ of the target $=$ $3.0 \mathrm{~mm}$. Again we assume two different cases for the diagnostic beam, a low intensity uranium $1 \mathrm{GeV} / \mathrm{u}$ beam and a low intensity $1.095 \mathrm{GeV} / \mathrm{u}$ argon beam, respectively. In Fig. 28 we plot the line density along the cylinder axis and the fraction of the energy carried out of the target by the uranium as well as the argon particles. It is seen that initially the line density along the axis is zero because the cylinder is hollow and therefore the particles of the diagnostic beam traveling along the cylinder axis pass through the target without any loss of energy. However, at about $t=135 \mathrm{~ns}$, the heated target material collapses at the axis and the line density along the target axis suddenly becomes $28 \mathrm{~g} / \mathrm{cm}^{3}$. The uranium particles are completely stopped in the target at this time.

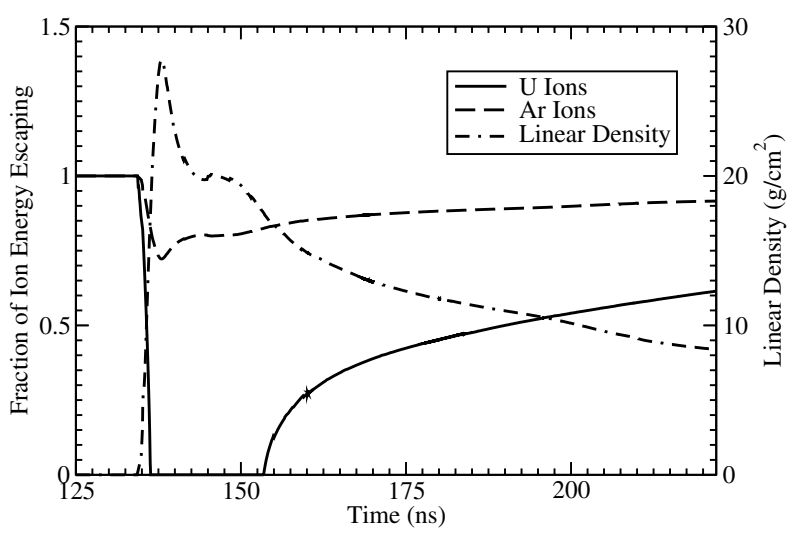

FIG. 28. Same as in Fig. 26, but using the beam-target geometry shown in Fig. 4(c).

At about $t=155 \mathrm{~ns}$, the particles start to reemerge from the target and the particle energy increases from zero to about $60 \%$ at $t=225 \mathrm{~ns}$, because the line density along the axis decreases due to the target expansion along the radial direction.

The argon ions have a much longer range than the uranium ions and they show a reduction in the escaping energy from $100 \%$ to about $70 \%$ at the time of maximum compression.

\section{A DISCUSSION OF DIAGNOSTIC METHODS}

The success and usefulness of an experiment is closely related to the ability of the experimentalists to diagnose the sample material. However this is not an easy problem and one needs to design and develop new diagnostic tools for the future experiments discussed in this paper. Since our simulation study predicts quantitatively the physical conditions (density, temperature, and pressure) in the beam-heated compressed material, we believe that this study will be very helpful to design and develop the appropriate diagnostic tools for the future experiments. In these experiments one needs to employ a complete set of diagnostic tools to measure the sample physical quantities.

Piezoelectric polymer stress gauges (pressure sensors made of polyvinildenfloride) immersed in the solid target may be applied to measure the pressures up to $0.1 \mathrm{Mbar}$. For higher pressures, Manganin pressure sensors could be used. However, due to technical difficulties, it is unlikely that one may be able to measure the value of pressure with a high enough accuracy. On the other hand, temperature, density profiles, shock velocity, and shock gradient may be measured accurately, as discussed below.

As an example, we consider the case of an elliptic focal spot in which different temperature and pressure profiles are created along the $x$ and the $y$ directions (see Figs. 21-25). In this case the shock velocity not only 
varies with time along the radius, but is also different in the $x$ and the $y$ directions. Since the target is fairly uniformly heated along the $z$ axis, the shock velocity and amplitude gradients can effectively be determined by shaping the target, for example, into stepwise uniform cylinders with different radii and measuring the arrival time of the shock/heat wave, most reasonably by optical means. In the following we consider two cylinders having radii 2 and $2.5 \mathrm{~mm}$, respectively, and that are observed from both the $x$ and the $y$ directions. It is seen from Fig. 21, that immediately after the end of the pulse $(20 \mathrm{~ns})$, the lead surface in the $y$ direction at $2.0 \mathrm{~mm}$ will start to expand due to the induced pressure in the Mbar range. The surface expansion can be observed by shadowgraphy, however the temperature will be too low to be detected. No change on the surface in the $x$ direction will be observed at this time.

During the following $150 \mathrm{~ns}$, the temperature smoothly increases to about $1 \mathrm{eV}$ in the $y$ direction (Fig. 23), which should be easily detectable on a free surface. However some material ablation had already occurred; the path of view may be blocked by cold vapor even if a transparent shield such as high-pressure He gas is used. One would therefore require additional opacity calculations together with hydrodynamic simulations in order to clarify what is to be expected.

Nothing will be detected in the $x$ direction until $t=$ $230 \mathrm{~ns}$ when a shock wave with about 2 Mbar pressure hits the surface of the target with a shock velocity of the order of $6 \mathrm{~km} / \mathrm{s}$. Since these parameters are quite similar to that in explosively driven shock wave experiments [27], a well-established means of optical diagnostics can be applied here. In the present parameter regime, "grey-body Plankian" radiation can be assumed for the optical range for most of the targets, including even rare gas crystals [28]. However, since the maximum of the radiation curve for a $\mathrm{kT}=1 \mathrm{eV}$ surface is situated below $250 \mathrm{~nm}$, a time and wavelength dependent recording of a wide spectral range, preferably UV and VUV, is recommended [29]. In particular the time history of the temperature increase will be a good indicator for the steepness of the shock front and thus for the pressure gradient induced asymmetrically by the ion beam. While there will be a steady increase in the intensity over the time in the $y$ direction, the emission in the $x$ direction will increase from zero to $100 \mathrm{~W} /\left(\mathrm{cm}^{2} \mathrm{~nm} \mathrm{sr}\right.$ ) (roughly the maximum of $1 \mathrm{eV}$ black body) in less than $10 \mathrm{~ns}$. Therefore a time resolution of the detector of about $2 \mathrm{~ns}$ has to be ensured. Moreover, there will be a gradual transition from the $x$ to the $y$ direction; a very high spatial resolution of less than $0.1 \mathrm{~nm}$ has to be achieved. One can use thin glass fibers for spatial definition and light transport which is preferable due to low cost and high flexibility. Shock velocity can then be easily measured by observing the light emission from the surface at different radii.
In addition to the surface light emission, the density profile can also be monitored. One way to measure the density profile in a cylindrically symmetric target is $\mathrm{x}$-ray absorption perpendicular to the ion beam axis and then carrying out an Abel inversion of the recorded signal. If one uses an elliptic beam focal spot, at least two pictures have to be taken in this case, one in the $x$ direction and the other in the $y$ direction. In this case at least $100 \mathrm{keV}$ photons have to be used in order to get reasonable transmission through a $5 \mathrm{~mm}$ thickness of lead. Although a beam line of the Petawatt laser facility, PHELIX, will be installed at the heavy ion beam-target area, most likely there will be only one focal spot to produce $100 \mathrm{keV}$ x rays. One can therefore use this facility to diagnose a target that is irradiated with a circular focal spot. For the case of irradiation with an elliptic beam, two conventional pulsed $\mathrm{x}$-ray sources with approximately $200 \mathrm{keV}$ acceleration voltage shall be used. Such sources however deliver only ns duration $\mathrm{x}$-ray flashes, defining a good time resolution over a longer period of time. It may however be possible to apply a picket-fence pulse and receive a time evolution profile.

\section{CONCLUSIONS}

This paper briefly discusses the design of the future SIS100 facility including the possibility to accelerate two beams of different species or a single beam of the same species but having an appropriate time structure. In the former case one beam will be a highest intensity bunched beam which will heat the target while the second beam will be a much lower intensity unbunched beam which will be used for diagnostic purposes. In the latter scheme the beam would have a leading high intensity peak for heating the target which will be followed by a low intensity long tail that can be used for diagnostic purposes.

Using the beam parameters that will be available at this future facility, we have carried out extensive simulation studies of beam-target interaction using different beam-target geometries. These include a solid lead cylindrical target irradiated with a beam having a circular focal spot, a solid cylinder irradiated with a hollow beam which has a ring shape focal spot, and a hollow cylindrical target that is irradiated with a hollow beam. These simulations show that one may be able to generate strongly coupled (nonideal) plasmas using the future SIS100 beam.

We have also done simulations using two different models for the EOS of lead, namely, the SESAME data and a multiphase semiempirical data. The two models show reasonably good agreement. Calculations have also been done using an elliptic focal spot and it has been shown that the pressure, density, and temperature profiles are very different along the semimajor and the semiminor axes of the ellipse. This information we believe will be 
very helpful for designing the future experiments on the studies of the thermophysical properties of HED matter including strongly coupled plasmas.

We also carried out calculations using two beams simultaneously. The energy of the particles of the imaging beam escaping along the target axis has been calculated for different beam-target geometries shown in Fig. 4. Such a curve can be measured in an experiment and a comparison of the experimentally obtained energy curve with the simulation results will allow one to check the validity of the EOS model [5].

\section{ACKNOWLEDGMENTS}

The authors would like to thank Professor C. Deutsch for many useful discussions. The authors also wish to thank the German Ministry of Research and Development (BMBF) for providing financial support to carry out this work.

[1] D. H. H. Hoffmann, K. Weyrich, H. Wahl, D. Gardes, R. Bimbot, and C. Fleurier, Phys. Rev. A 42, 2313 (1990).

[2] S. Stöwe, R. Bock, M. Dornik, P. Spiller, M. Stetter, V. E. Fortov, V. Mintsev, M. Kulish, A. Shutov, V. Yakushev, B. Sharkov, A. Golubev, B. Bruynetkin, U. Funk, M. Geissel, D. H. H. Hoffmann, and N. A. Tahir, Nucl. Instrum. Methods Phys. Res., Sect. A 415, 384 (1998).

[3] U. Funk, R. Bock, M. Dornik, M. Geissel, M. Stetter, S. Stöwe, N. A. Tahir, and D. H. H. Hoffmann, Nucl. Instrum. Methods Phys. Res., Sect. A 415, 68 (1998).

[4] U. Neuner, R. Bock, M. Roth, P. Spiller, C. Constantin, U. N. Funk, M. Geissel, S. Hakuli, D. H. H. Hoffmann, J. Jacoby, A. Kozyreva, N. A. Tahir, S. Udrea, D. Varentsov, and A. Tauschwitz, Phys. Rev. Lett. 85, 4518 (2000).

[5] D. Varentsov, P. Spiller, U. N. Funk, D. H. H. Hoffmann, A. Kozyreva, N. A. Tahir, C. Constantin, E. Dewald, J. Jacoby, U. Neuner, S. Udrea, and R. Bock, Nucl. Instrum. Methods Phys. Res., Sect. B 174, 215 (2001).

[6] V. E. Fortov et al., Nucl. Sci. Eng. 123, 169 (1996).

[7] N. A. Tahir et al., Phys. Rev. E 61, 1975 (2000).

[8] N. A. Tahir et al., Phys. Rev. E 62, 1224 (2000).

[9] N. A. Tahir et al., Phys. Rev. E 63, 016402 (2001).

[10] I. Hofmann, O. Boine-Frankenheim, R.W. Hasse, and P. Spiller, GSI-Report No. GSI-2001-4, 29, 2001.
[11] P. Spiller et al., in Proceedings of the PAC1999, New York (IEEE, Piscataway, NJ, 1999), p. 1788.

[12] J. F. Ziegler, J. P. Biersack, and U. Littmark, The Stopping and Ranges of Ions in Solids (Pergamon Press, New York, 1996).

[13] A. V. Bushman and V. Fortov, Sov. Technol. Rev. B 1 (1987).

[14] I.V. Lomonosov, A.V. Bushman, and V. E. Fortov, in High-Pressure Science and Technology, edited by S.C. Schmidt, J.W. Schaner, G. A. Samara, and M. Ross (AIP Press, New York, 1994), Pt. 1, p. 117; C. Narayan, H. Luo, J. Orlof, and A. L. Ruoff, Nature (London) 393, 46 (1998).

[15] H. K. Mao and R. J. Hemley, Rev. Mod. Phys. 66, 671 (1994).

[16] S.T. Weir, A. C. Mitchell, and W. J. Nellis, Phys. Rev. Lett. 76, 1860 (1996).

[17] W. J. Nellis, A.C. Mitchell, P.C. McCandless, D. J. Erskine, and S. T. Weir, Phys. Rev. Lett. 68, 2937 (1992).

[18] V. E. Fortov, V. Ya. Ternovoi, S. V. Kvitov, V. B. Mintsev, D. N. Nikolaev, A. A. Pyalling, and A.S. Filimonov, JETP Lett. 69, 926 (1999).

[19] V. Ya. Ternovoi, A. S. Filiminov, V. E. Fortov, S. V. Kvitov, D. N. Nikolaev, and A. A. Pyalling, Physica (Amsterdam) 265B, 6 (1999).

[20] P. M. Celliers, G.W. Collins, L. B. Da Silva, D. M. Gold, R. Cauble, R. J. Wallace, M. E. Foord, and B. A. Hammel, Phys. Rev. Lett. 84, 5564 (2000).

[21] R. Cauble, P. M. Celliers, G.W. Collins, L. B. Da Silva, D. M. Gold, M. E. Foord, K. S. Budil, R. J. Wallace, and A. Ng, Astrophys. J. 127, 267 (2000).

[22] M. D. Knudson et al., Phys. Rev. Lett. 87, 225501 (2001).

[23] R. Cauble, D. W. Phillion, T. J. Hoover, N. C. Holmes, J. D. Kilkenny, and R.W. Lee, Phys. Rev. Lett. 70, 2102 (1993).

[24] D. H. H. Hoffmann, V. E. Fortov, I.V. Lomonosov, V. Mintsev, N. A. Tahir, D. Varentsov, and J. Wieser, Phys. Plasmas 9, 3651 (2002).

[25] G. I. Kerley, Los Alamos Scientific Laboratory Report No. LA-4776, UC-34, available at Technical Information Service, U.S. Department of Commerce, 6285 Port Royal Road, Springfield, Virginia 22151.

[26] N. A. Tahir et al., Nucl. Instrum. Methods Phys. Res., Sect. B (to be published).

[27] S.V. Kvitov et al., JETP Lett. 53, 353 (1991).

[28] F.V. Grigor et al., Sov. Phys. JETP 61, 751 (1985).

[29] H. B. Radouskiy and M. Ross, Phys. Lett. A 129, 43 (1988). 